\title{
Salvaging an entrapped hand unusual injury, unusual management
}

\section{Introduction}

Hand injuries account for $10 \%$ of emergency visits to the hospital. ${ }^{1}$ Injuries to the hand occur due to a wide variety of causes..$^{2-4}$ The hand is equipped to carry out prehensile function and is placed in various positions in the space to carry out different functions by gripping, hooking or grasping. As a consequence of this the hand has a propensity to get injured easily and frequently. Our case reported with a hand which was trapped within a mechanized meat grinder. The case report highlights the unusual and quick decision making that led to salvage of a large portion of the hand. Out of the box thinking can help in such situations when trauma management protocols do not provide answers and equipment for management.

\section{Case report}

A 29 year old male reported to the casualty ward of our hospital with his hand entrapped within the meat mincer. He had been feeding the hopper of the mincer with his hands and during this process, his hand had got trapped between the rotating and the stationary parts of the machine. His associates disconnected the part of the machine from the main motor and its attachments and travelled several kilometers to the hospital with the hand being entrapped within the mincer.

At casualty reception, the patient was administered analgesics and antibiotics. On assessing the injury carefully, it was decided that reversing the machine would crush the hand further. The thickness of the machine made the likelihood of cutting the machine apart, with tools that could be found in the hospital, unlikely. The only plausible way of freeing the hand seemed to be amputation of the fingers which was not easy due to the problems of accessing the metacarpophalangeal joint areas through the narrow mouth of the hopper. A departmental meeting was called to discuss the emergent nature of the case. It was suggested that help from a car workshop within the vicinity be sought. The mechanics at the car workshop felt that they could cut open the mincer if the patient could be taken to the shop floor.

A supraclavicular block was administered and the patient was sent to the car workshop with a support team of anaesthetists and orthopaedicians. The mechanics at the workshop used a body cutting high speed rotating saw to pry open the machine carefully. While the saw was being used, water was sprinkled onto the mincer to prevent thermal injury. The hand was freed. However, the middle and distal phalanges of the ring finger of the hand could not be salvaged. Fractures in the other fingers were stabilized by means of $\mathrm{k}$ wires. 3 weeks postoperatively, the patient's rehabilitation is proceeding well.

\section{Discussion}

Hand injuries are often sustained at the workplace. ${ }^{5}$ These injuries also occur at home, in road traffic incidents and with large grinding machines. ${ }^{4}$ Injection injuries have been reported where despite optimal care, amputation and dysfunction rates with high-risk injection injuries
Volume 7 Issue 6 - 2017

\author{
Saheel Maajid, Tarsem Lal,Towseef Ahmed \\ Bhat, Furqan Mir, Sheikh Sarvar, Shabir \\ Ahmed Dhar, Zameer Ali \\ SKIMS Medical College \& Hospital, India
}

Correspondence: habir A Dhar, The Skims Mc Bemina, Srinagar, Kashmir 190017, India, Email shabirdhar@yahoo.co.in

Received: January 31, 2017 | Published: April 13, 2017

(due to higher psi, organic solvents, and/or delayed definitive care) have been reported from $48 \%$ to $80 \%,{ }^{6,7}$ Penetrating injuries of the hand are generally encountered in cases of gunshot and stab injuries.

The integument of the hand is easily damaged and open fractures are quite common. Clindamycin and bacitrim are useful for MRSA. Aminoglycosides are added for contaminated wounds and penicillin for farm equipment. However often the hands injured in machinery accidents are non-salvageable due to massive crushing or, as in our case, difficulty in retrieving the hand from the entrapped position within a machine.

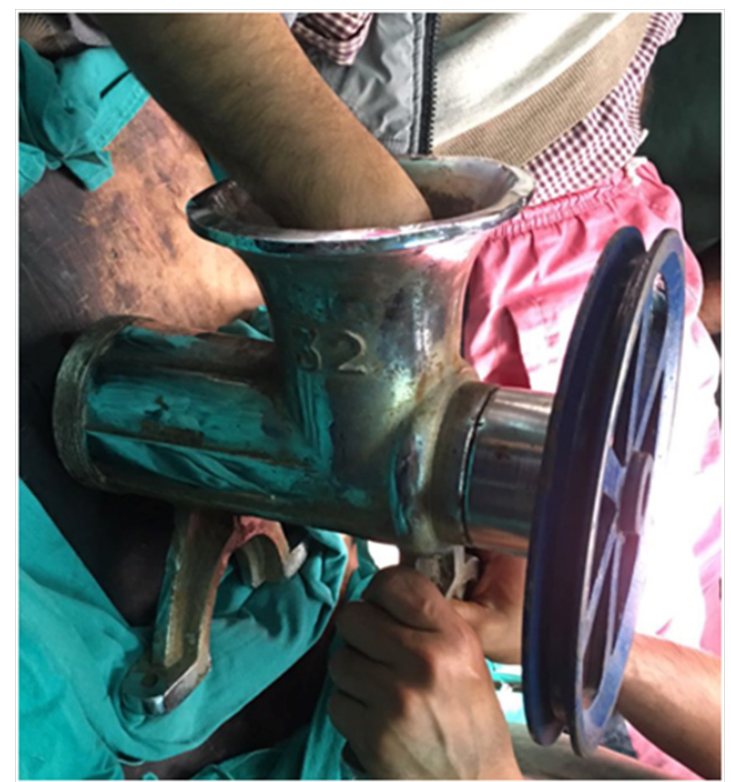

Figure I The hand within the mincer.

Our case provided a challenge to the casualty team. Before we could get to the management of the injury itself, we had to get the hand out of the machine. This called for an unusual solution as the hospital emergency department was not equipped with cutting 
equipment and neither did we have the requisite expertise to handle such machinery. It was decided to take the patient to the car workshop whilst maintaining the theatre essentials within an ambulance. The machine was successfully cut apart and a major portion of the hand was salvaged. This case highlights the need to think laterally in unusual situations in the casualty management of patients (Figures $1-5)$.

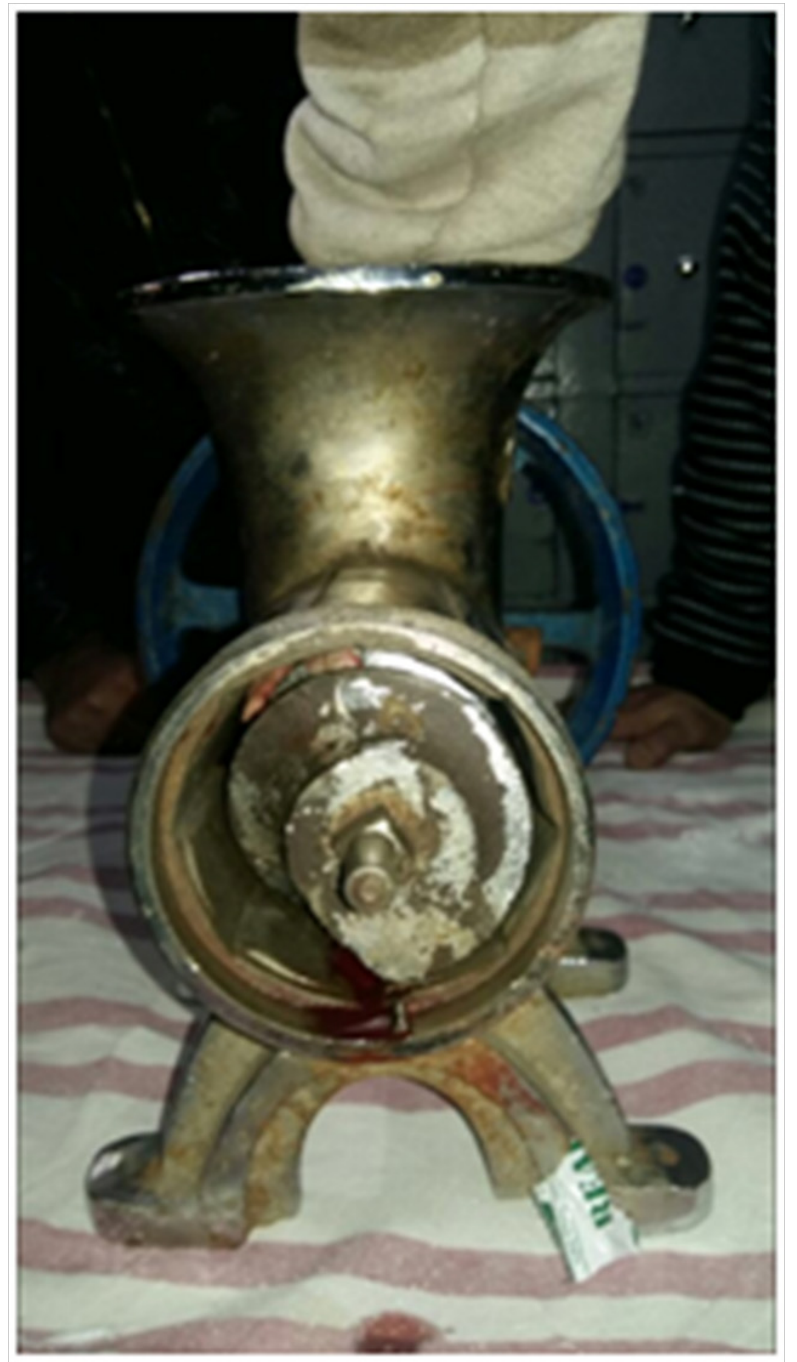

Figure 2 The fingers can be seen entrapped.

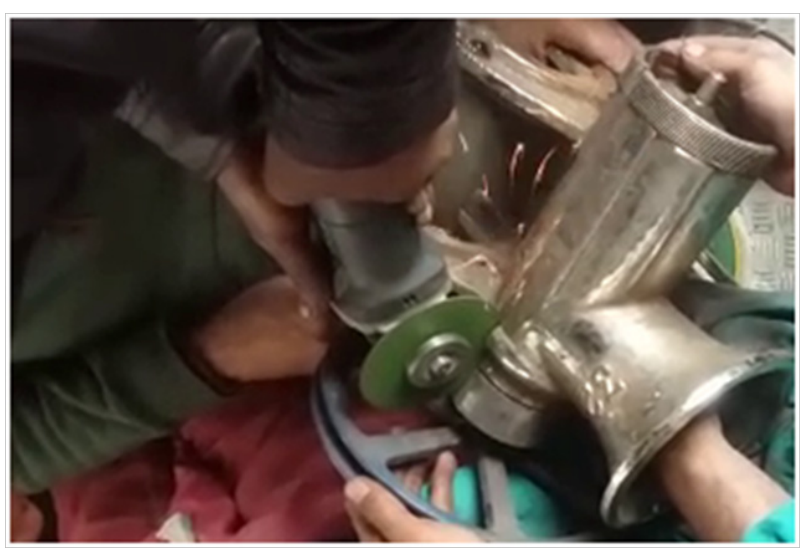

Figure 3 The cutting machine being used on the mincer.

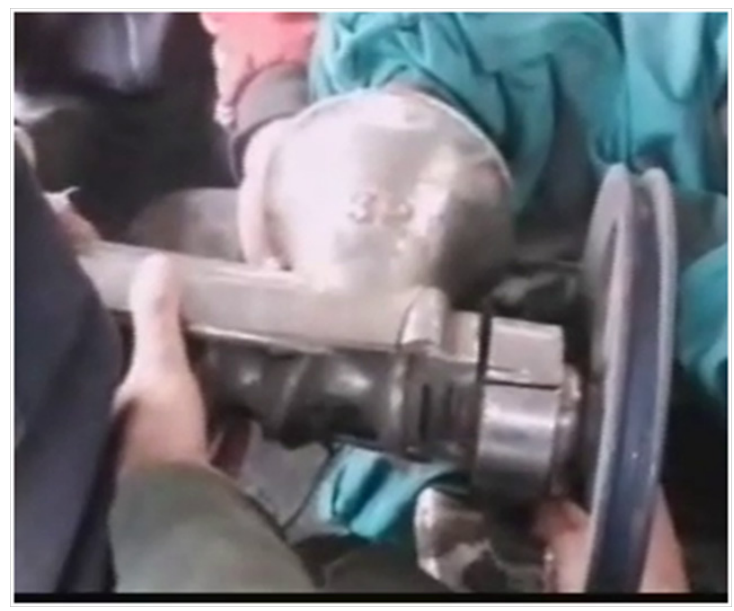

Figure 4 The macine being pulled apart.

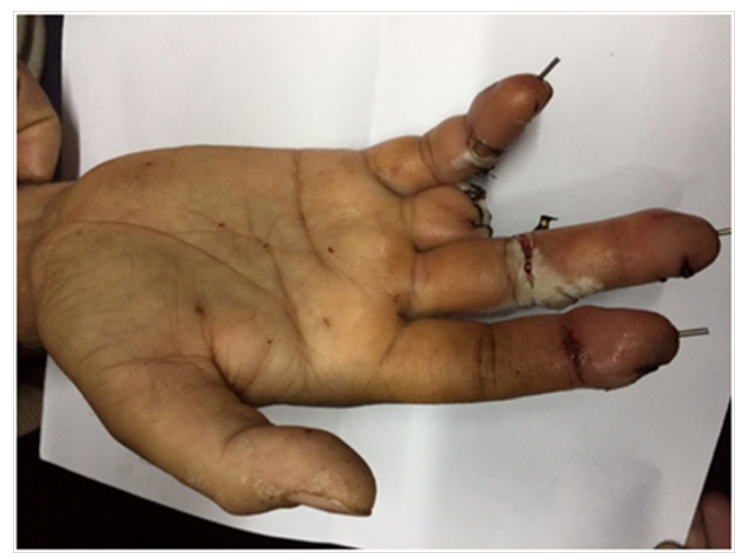

Figure $\mathbf{5}$ The salvaged hand.

\section{Acknowledgments}

None.

\section{Conflicts of interest}

None.

\section{References}

1. Resnik CS. Wrist and hand injuries. Semin Musculoskelet Radiol. 2000;2:193-204.

2. Beaton AA, Williams L, Moseley LG. Handedness and hand injuries. $J$ Hand Surg. 1994;19(2):158-161.

3. Hollis JL, Watson DP. The relationship between handedness, mechanism of injury and which hand injured. J Hand Surg. 1993;18(3):394.

4. Adigun IA, Kuranga SA, Abdulrahman LO. Grinding machine: Friend or foe. WAJM. 2002;21(4):338-340.

5. I Adigun, K Ogundipe, A Aderibigbe. Pattern of Hand Injuries In A Teaching Hospital Of A Developing Country: A Three Year Review Of Cases. The Internet Journal of Hand Surgery. 2006;1(1):1-6.

6. Mirzayan R, Schnall SB, Chon JH, et al. Culture results and amputation rates in high-pressure paint gun injuries of the hand. Orthopedics. 2001;24(6):587-589.

7. Wong TC, Ip FK, Wu WC. High-pressure injection injuries of the hand in a Chinese population. J Hand Surg Br. 200530(6):588-592. 